$\mathbb{P}$ periodica polytechnica

Civil Engineering

$54 / 2(2010) 73478$

doi: 10.3311/pp.ci.2010-2.01

web: http://www.pp.bme.hu/ci

(c) Periodica Polytechnica 2010

RESEARCH ARTICLE

\title{
Optimal suspension settings for ride comfort of road vehicles
}

\author{
Dezső Szőke / János Lógó / Dániel B. Merczel
}

Received 2010-11-08, accepted 2010-11-29

\begin{abstract}
In this paper a numerical study is presented for the effect of the settings of the suspension parameters of a road vehicle for the ride comfort. The chassis of the IK GMC midibus model is investigated as an example. The suspension parameters are represented by hard springs and dampers. By the appropriate choice of the suspension parameters it can be achieved that the influence of the dynamical forces is minimal for the oscillation of the carbody and for the dynamically computed internal forces, respectively. The nonlinear constrained optimization models provide appropriate tools to investigate the effect of the suspension parameters for the average acceleration and displacement of the chassis, respectively.
\end{abstract}

\section{Keywords}

chassis $\cdot$ suspension parameters $\cdot$ stochastic loading $\cdot$ mathematical programming $\cdot$ sensitivity analysis

\section{Acknowledgement}

This work is connected to the scientific program of the "Development of quality-oriented and harmonized $R+D+I$ strategy and functional model at BME” project. This project is supported by the New Hungary Development Plan (Project ID: TÁMOP4.2.1/B-09/1/KMR-2010-0002).

The present study was supported by The Hungarian National Scientific and Research Foundation (OTKA T 81185).

\section{Dezső Szőke}

Department of Vehicles Chassis and Light Weight Structures, BME, H-1111 Bertalan Lajos u. 2, Budapest, Hungary

e-mail: dezso@kme.bme.hu

\section{János Lógó}

Department of Structural Mechanics,BME, H-1111 Múegyetem rkp 3, Budapest, Hungary

e-mail: logo@ep-mech.me.bme.hu

\section{Dániel B. Merczel}

Department of Structural Mechanics, BME, H-1111 Múegyetem rkp 3, Budapest, Hungary

e-mail: dmerczel@mail.hu

\section{Introduction}

The dynamic analysis, optimization and its tools play a very important aspect in all fields of vehicles and traffic research [1, 2, 8, 11, 16]. The settings of the suspension parameters of commercial vehicles are a very intensively investigated topic [17]. A special study was presented in [3] where a large variety of studies and up-to-date techniques were developed for vehicle seats used by different types of transportation system such as cars, trucks, tractors, trains and aircrafts. Computational procedures and optimization of the dynamic behaviour of passively or actively suspended road vehicles are investigated in [4, 5, 7, 9, 12].

The cushioning material in a vehicle seat plays a dominant role in supporting operator posture, isolating vibration and improving ride quality. Vibration attenuation in a tractor seat is achieved by selecting proper suspension and damping mechanism. Several commercially available seat cushion materials of different densities, thickness and compositions were studied in [6].

This paper is a revised and extended version of the VSDIA Conference presentation of Szôke et al. [15]. The aim of this paper is to present a numerical model and computational procedure for the effect of the settings of the suspension parameters of a midibus for the ride comfort. The carbody/chassis of a vehicle is supported through the elastic components (hard springs, dampers, tires) of the axle/wheel suspension system on the irregular surface of the road. The irregular road surface causes time dependent kinematical loading process [10,14] during the motion of the vehicle. By the appropriate choice of the suspension parameters it can be achieved that the influence of these forces is minimal for the oscillation of the carbody and for the dynamically computed internal forces, respectively.

The estimation of the optimum parameters is presented by using the FEM model of a midibus. The solution to the problem requires a very effective analytical computational procedure which is capable to compute the dynamical parameters (acceleration of the oscillation, internal forces, stresses) of the thousands degree of freedom structure on the one hand and the objective function can be generated by using of these parameters, on the 
other. Moreover an optimization algorithm is also necessary for the constrained optimization.

In the case of a given vehicle or its numerical model the type of road, the pay load and the speed of the vehicle are variables, which fundamentally influence the parameters of the structural dynamics of the vehicle considered. These types of operational loading are described by a stochastic process [14]. In this way it is advised to use sensitivity analysis, which concentrates on the effect of system parameters (structural model, loading) concerning the sensitivity of the objective function.

\section{Problem determination}

\subsection{Requirements of the suspension system}

The elastic components of the suspension of a vehicle are: tires, hard springs and dampers. These elements have well defined characteristics (usually they are given by linearized parameters) in the case of a given type of vehicle. The irregular road surface causes time dependent stochastic kinematical loading (extended by accidental loading, such as an intersection with rail roads) which depends on the statistical characteristic (surface type, material, etc.) of the road and the speed of the vehicle. In case of commercial vehicles (trucks, buses) the payload can be varied in a significant interval. Due to this fact the elements of the suspension system have to be loading dependent controlled (air-springs). Controlled active or semi-active suspension systems can be applied if there are altering circumstances and requirements, as well. Unfortunately, they can be used only in research projects due to the high cost and energy requirement. In that case the applied mechanical model of the vehicles has low degree of freedom (a quarter of the vehicle is considered) and the discrete road effects (intersection with rail roads) are not considered fully [7, 9].

There is no energy demand if the characteristics of the suspension systems are changed according to the parameters (speed, type of the road, pay load) of the stochastically altering excitation to reach the optimal oscillations (comfort, stability). Application of dampers and air-springs (opening or closing valves) are the most suitable practical solution in that case. The numerical interpretation of these mechanical models are more complex: $2 \mathrm{D}$ or $3 \mathrm{D}$ rigid-body models, elastic beam model of the chassis are applied with the extension adaptive controllers [12,13].

The recent FEM models of the vehicles have thousands of degree of freedom in dynamical calculations and there is a demand to determine the "optimal" characteristics of the suspension system.

In the following the estimation of the optimal parameters of the suspension system is presented by use of a FEM model of a midibus. The numerical model consists of two main parts: an analytical one for the calculation of the structural dynamics quantities (accelerations, internal forces, stresses,...) and a constrained optimization part where the objective function is generated by the use of the computed quantities.

\subsection{Mechanical and mathematical modellization}

The well-known procedures to road profile simulations in general use the real coherence function instead of the complex cross-spectra, consequently the simulated autospectrum function and standard deviation approach are only the target ones. The first author presented a method where the above-mentioned procedures are improved by the consideration of the nature of these cross-spectral characteristics [14]. As a result, the simulated tracks computed by the improved procedure represent exactly the target standard deviation and autospectrum function. The applicability and effectiveness of the discussed procedure were demonstrated by the analysis of measured data of a Hungarian highway. In the following this type of kinematic loading is considered [14]. Neglecting the details the road profile realisations of the two tracks with the assumptions that the rear wheels exactly follow the front ones can be determined as follows:

$$
x_{1}(t)=\Delta f\left(T^{\frac{1}{2}}\right) \sum_{k=0}^{N-1} G\left(f_{k}\right)^{\frac{1}{2}} e^{i\left(2 \pi f_{k} t+\varphi_{1}\left(f_{k}\right)\right)}
$$

and

$$
x_{2}(t)=\Delta f\left(T^{\frac{1}{2}}\right) \sum_{k=0}^{N-1} G\left(f_{k}\right)^{\frac{1}{2}} e^{i\left(2 \pi f_{k} t+\varphi_{1}\left(f_{k}\right)\right)} c e^{i \beta} .
$$

Here $G(f)$ is the spectral matrix of the tracks and the crossspectra is represented by its off diagonal elements. $\gamma(f)$ is the coherence function. $\beta(f)$ is the phase angle and i denotes the imaginary unit. $T=(N-1) \Delta t$, where $\Delta t=1 / T, f_{k}=k \Delta f$, $k=0,1,2, \ldots, N-1,0 \leq t \leq T, \phi 1\left(f_{k}\right)$, independent random variable (phase angle) uniformly distributed between 0 and $\pi$. $\Delta t$ is chosen by the consideration of the Nyquist frequency, that is $f N y=1 /(2 \Delta t)$. $C$ is a calculated value, but very often it is $1[14]$.

As it is mentioned a FEM model of a midibus (IK GMC model) is used to demonstrate the numerical procedure. The model can be seen in Fig. 11. The tires, the hard springs and the dampers are substituted by appropriately linearized spring and damper elements, respectively. The internal damping of the chassis is assumed to be proportional with the rigidity of the carbody [13]. The speed of the vehicle and the road type are given. The analysis is based on the following equation system:

$$
\mathbf{M} \cdot \ddot{\mathbf{z}}(t)+\mathbf{C} \cdot \dot{\mathbf{z}}(t)+\mathbf{K} \cdot \mathbf{z}(t)=\mathbf{F}(t)
$$

Here $\mathbf{M}, \mathbf{C}$ and $\mathbf{K}$ are the mass, damping and stiffness matrices of the midibus model, respectively. The external load due to the kinematical effects is denoted by $\mathbf{F}(t)$ and $z(t)$ is the unknown vector of the nodal displacements.

By the use of the solution (absolute or relative displacement of given points) of Eq. (3) different physical quantities (velocities, accelerations, stresses,...) can be derived as statistical response of the structure what are used to construct the objective function of the optimization procedure. Among the elements of 
Mechanical model, Assumptions

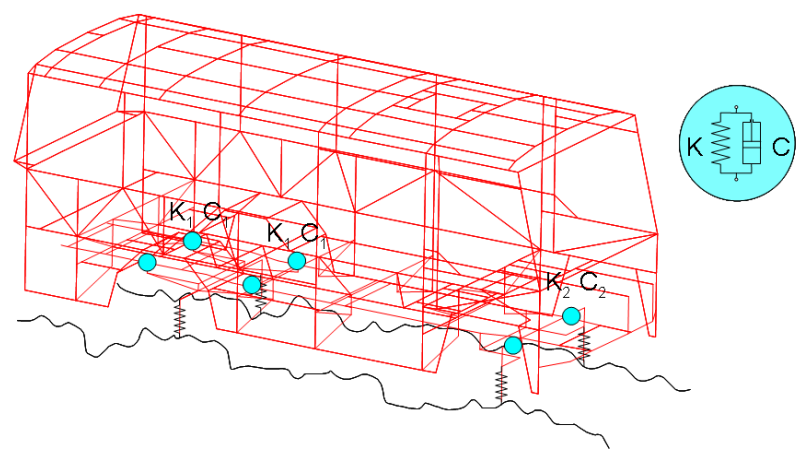

Fig. 1. FEM model of IK GMC midibus

the suspension system the characteristics of the hardsprings and the hydraulic dampers can be altered in the easiest way. Since opening or closing valves are the practical solution in case of vehicles to alter the rigidity of the hardsprings and damping coefficients it is enough to find the optimal characteristics of these parameters (in our problem it means to find 2.2 parameter values ( $K_{1}, C_{1}, K_{2}, C_{2}$ in Fig. 1). Because it is advised that these parameters should be in a given range ( $40 \%$ of the factory values) a constrained mathematical programming problem has to be solved. Structural dynamics point of view the acceleration of the chassis and its variation are the best quantities which can be used to approximate the comfort feeling of the passengers and the dynamical internal forces. In this way the mathematical programming problem can be constructed as follows:

$$
E\left\{D\left(\ddot{z}_{i}\right)_{i=1, \cdots, N}\right\} \rightarrow \min !
$$

with altering the values of the dampings and rigidities, respectively.

$$
d C_{j}= \pm 0.4 C_{;} \quad d K_{j}= \pm 0.4 K_{j} ; \quad j=1, \cdots, m
$$

Here the objective function $E\left\{D\left(\dot{z}_{i}\right)_{i=1, \cdots, N}\right\}$ means the average of the variance of the acceleration of the nodes of the FEM model of the chassis, $N$ is number of the nodes, $m$ is the number of the suspensions.

\section{Numerical example}

On the basis of our theoretical investigations and numerical simulations the following FEM model (Fig. 1) and loading were considered:

- 325 nodes, 550 finite elements (beam and shell elements),

- speed: $10 \mathrm{~m} / \mathrm{s}$,

- type of the road: asphalt with $1 \mathrm{~cm}$ variance.

To construct the objective function in Eq. (4) the acceleration spectrums (PSD) of the nodes have to be computed. Modal condensation is applied with discrete damping elements in the numerical procedure [13]. By the help of this method the computational time of the construction of the objective function does not depend on the number of the nodes significantly. To speed-up the numerical calculation a sensitivity analysis is advised. Here a simplified investigation was performed to examine the change of the objective function due to the variation of the 4 suspension parameters. The sensitivity function is constructed in a way to allow $10 \%$ change of the suspension parameters $\left(K_{1}, C_{1}, K_{2}\right.$, $\left.C_{2}\right)$ which makes a surface consisting of $9^{4}(\approx 6600)$ points. The CPU time is cc. 80 minutes on a $\mathrm{PC}(\mathrm{P} 4$ processor) using the MATHLAB software.

The variation of the nodal accelerations due to the change of the suspension parameters can be seen in Fig. 2 (acceleration sensitivity).

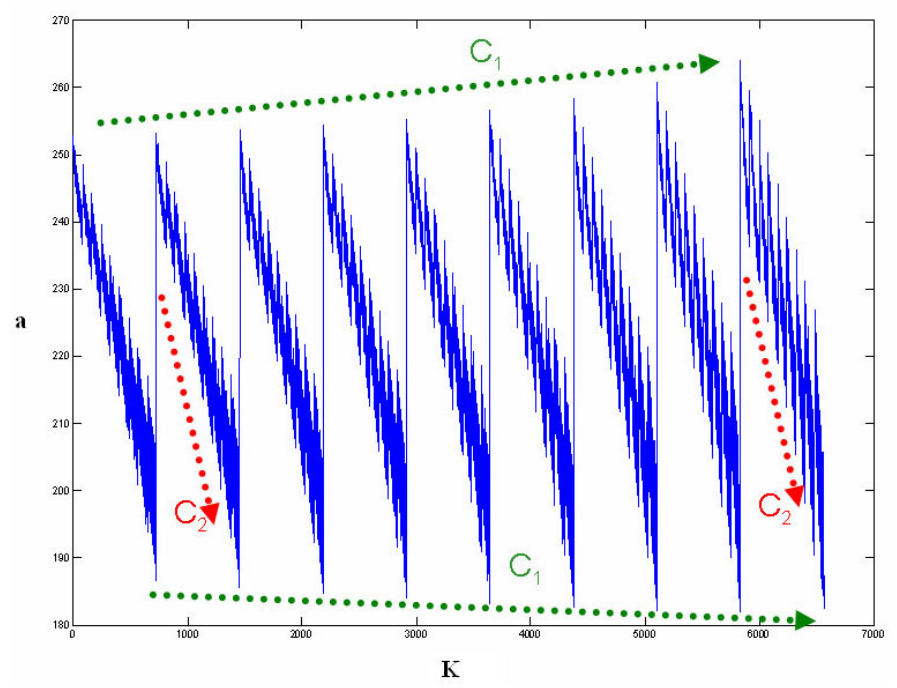

Fig. 2. Variation of the accelerations

One can see that:

- the influence of the rear damper $\left(C_{2}\right)$ is the most significant one and the effect is unambiguous,

- the influence of the front damper $\left(C_{1}\right)$ is less significant and the trend is variable,

- the "optimal" parameter values are located at the border of the given domain (or near),

- the objective function is rather flat which can make difficulties during the optimization procedure.

Same conclusions can be obtained if the variation of the nodal displacements due to the change of the suspension parameters are pictured (Fig. 3 displacement sensitivity).

- Naturally the influence of the dampings is the opposite.

- There are several methods to conduct the optimization procedure and here the OPTIM toolbox of the MATHLAB is used with applying the FMINCON and FMINIMAX algorithms. Both procedures lead to the same results and 5-6 or 8-9 major iterations are necessary for obtaining the optimal solution, respectively. These iteration numbers are significantly less than the $9^{4}$ steps during the sensitivity analysis. 


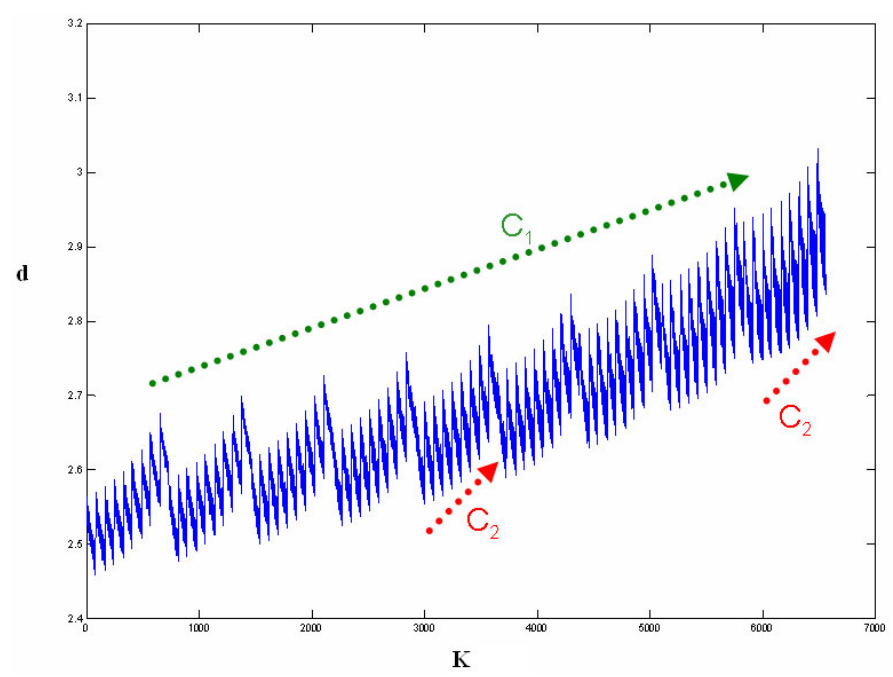

Fig. 3. Variation of the displacements



Fig. 4. Variation of the acceleration of the carbody without and with optimization



Fig. 5. Variation of the acceleration of the carbody by parametric cutting
The variation of the nodal accelerations of the carbody due to the change of the suspension parameters can be seen without and with optimization in Fig. 4. The design variables are the 4 suspension parameters. The optimal solution is the reference values with the following modifications of the factory settings: $d C_{1}=-0.3004 C_{1}, d C_{2}=-0.4000 C_{2}, d K_{1}=$ $-0.4000 K_{1}, d K_{2}=-0.4000 K_{2}$. Due to these modifications the average acceleration of the carbody is decreased by $18 \%$ $\left(f_{0}=222.6493 \mathrm{~cm} / \mathrm{s}^{2}, f_{\min }=182.0372 \mathrm{~cm} / \mathrm{s}^{2}\right)$. One can see in Fig. 4 that the variances of the nodal accelerations are decreased, as well. The applicability of the optimization procedures and algorithms can be proved by the fact that the optimal solution is in good agreement with the result (estimations are $f_{\text {est } 1}=182.2121 \mathrm{~cm} / \mathrm{s}^{2}$ and $f_{\text {est } 1}=182.2669 \mathrm{~cm} / \mathrm{s}^{2}-$ a part of the diagram can be seen in Fig. 5p of the parameter "cutting" method ( $9^{4}$ steps).

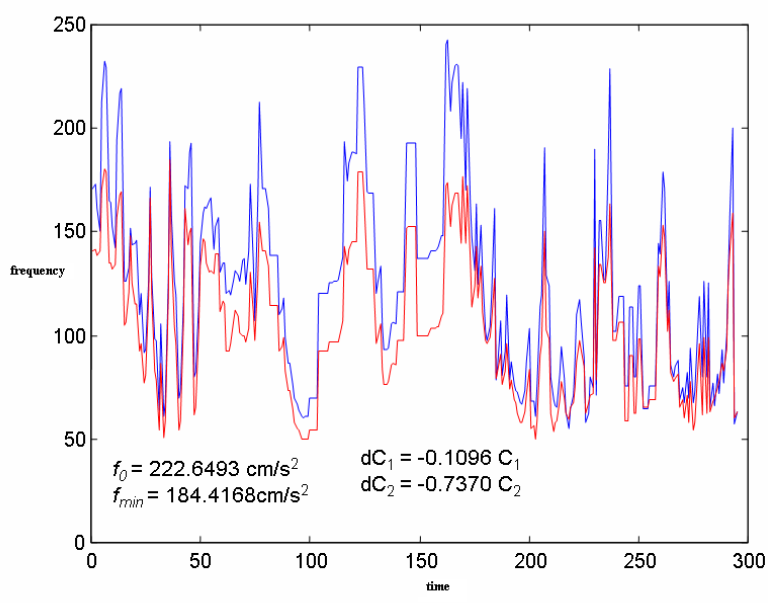

Fig. 6. Variation of the acceleration of the carbody (2 parameters)

It was investigated that only the two dampers are the design variables in the optimization procedure with the allowance of $d C_{i}=80 \%$ which are extreme damping. The variation of the nodal accelerations of the carbody due to the change of the characteristic of the dampers can be seen in Fig. 6 without and with optimization. The optimal solution is: $f_{\min }=184.4168 \mathrm{~cm} / \mathrm{s}^{2}$, $d C_{1}=-0.1096 C_{1}, d C_{2}=-0.7370 C_{2}$. One can see that the optimum of two parameters is near to the optimum of four parameters, but in this case the transient oscillation "answers" of the chassis are not damped sufficiently for the accidental surface problem which may cause decrease in the stability of the vehicle.

Theoretically it is possible to alter the internal damping of the chassis, as well. The optimal solution of this 5 parameter optimization problem can be seen in Fig. 7. The optimal solution is: $f_{\min }=164.4926 \mathrm{~cm} / \mathrm{s}^{2}, d C_{1}=-0.2062 C_{1}$, $d C_{2}=-0.4000 C_{2}, d K_{1}=-0.4000 K_{1}, d K_{2}=-0.4000 K_{2}$, $d \lambda=0.4000 \lambda$. These results confirm the applicability of the elastic beam model [13] which can be seen in Fig. 8 By increasing the internal damping the elastic carbody becomes more 
rigid which fundamentally determines (or may determine) the decreasing of the acceleration of the chassis.

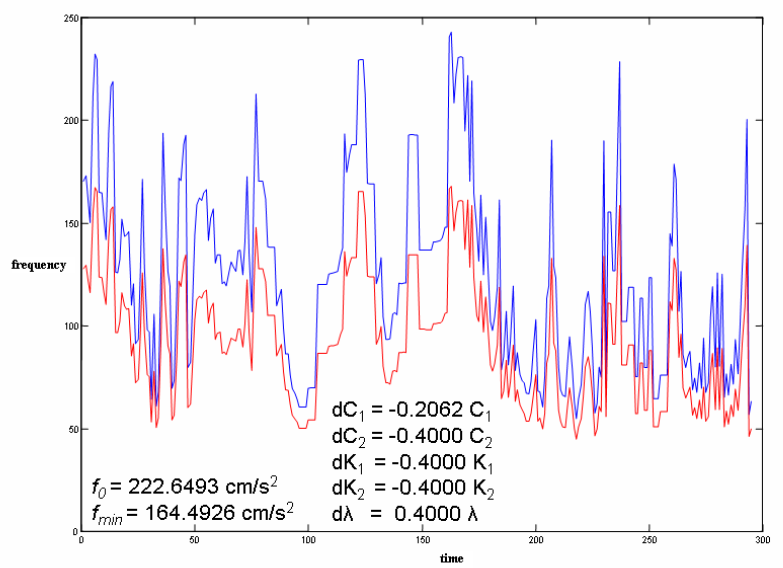

Fig. 7. Variation of the acceleration of the carbody (5 parameters)

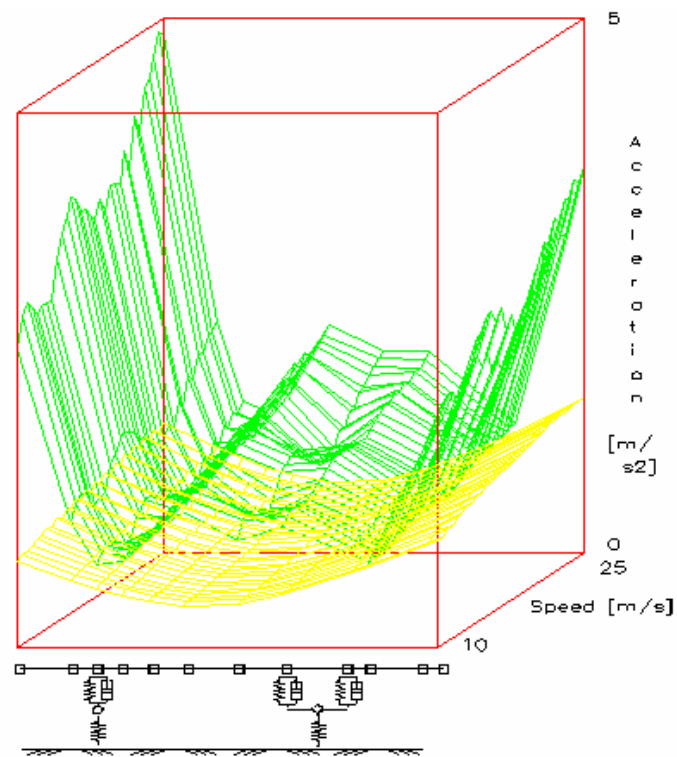

Fig. 8. Variation of the acceleration of the carbody in function of speed and length

With a small modification the vertical displacements of the nodes can be used as components of the objective function in problem (2). The optimal solution can be seen in Fig.9. One can see that the characteristic of the suspension parameters should be modified partly in different directions $\left(d C_{1}=0.4 C_{1}, d C_{2}=\right.$ $\left.0.4 C_{2}, d K_{1}=-0.4 K_{1}, d K_{2}=-0.4 K_{2}\right)$.

\section{Conclusion}

On the basis of our theoretical investigations and numerical simulations, the following conclusions can be drawn:

- the operation of the vehicle can be approximately described by using simplified stochastic model,

- the stochastically loaded structural optimization problem was solved,



Fig. 9. Variation of the displacements of the nodes

- the introduced algorithm provides an appropriate tool to estimate the optimal suspension parameters in the case of given loads with thousand degree of freedom,

- the algorithm is rather stable and provides the convergence to reach the optimum,

- the selection of the objective function fundamentally affects the optimal solution.

\section{References}

1 Els P. S., Uys P. E., Snyman J. A., Thoresson M. J., Gradient-based approximation methods applied to the optimal design of vehicle suspension systems using computational models with severe inherent noise, Mathematical Computer Modelling 43 (2006), 787-801, DOI 10.1.1.132.5544.

2 Eriksson P., Ride comfort optimization of buses, Chalmers Tekniska Högskola / Chalmers University of Technology, Göteborg, Sweden, 2002. $\mathrm{PhD}$ thesis.

3 Fai T. C., Delbressine F., Rauterberg M., Vehicle seat design: State of the art and recent development, World Engineering Congress 2007 ( Penang, Malaysia, 2007), 2007, pp. 51-61.

4 Gobbi M., Mastinu G., Analytical description and optimisation of the $d y-$ namic behaviour of passively suspended road vehicles, Journal of Sound and Vibration 245 (2001), no. 3, 457-481, DOI 10.1006/jsvi.2001.3591.

5 Kuti I., A computational procedure for nonlinear dynamic analysis of vehicles, Vehicle System Dynamics 30 (1998), no. 1, 37-54, DOI 10.1081/00423119808969434.

6 Mehta C. R., Tewari V. K., Damping characteristics of seat cushion materials for tractor ride comfort, Journal of Terramechanics 47 (2010), no. 6, 401-406, DOI 10.1016/j.jterra.2009.11.001.

7 Michelberger P, Bokor J, Palkovics L, Robust design of active suspension system, International Journal of Vehicle Design 14 (1994), no. 2/3, 145-165, DOI 10.1016/j.jterra.2009.11.001.

8 Mitschke M., Fahrzeugdynamik, B B, Springer Verlag, 1990.

9 Palkovics L, Bokor J, Verhovens P., Design problems of the semi-active wheel suspension system and a possible way of their elimination, XXV. FISITA congress (Beijing, 1994), 1994, pp. 30-40.

10 Peter T, Bellay A, Integral transformations of road profile excitation spectra for variable vehicle speeds, Vehicle System Dynamics 15 (1986), 19-40.

11 Snyman J A, Hay A M, The Dynamic- $Q$ optimisation method: an alternative to SQP?, Computers \& Mathematics with Applications 44 (2002), no. 12, 1589-1598, DOI 10.1016/S0898-1221(02)00281-X. 
12 Szöke D, Gajdar T, Optimal Control of Flexible Bus Structures, 28th ISATA Conference (Stuttgart, 1995), 1995, pp. 18-22.

13 Szöke D, Effect of non-linearities on the dynamic loads of bus framework structure, 30th Meeting of Bus and Coach Experts (Győr, 1999), 1999, pp. 247-257.

14 Szöke D, Kuti I, A new development in the numerical description of road profile realisations, International Journal of Vehicle Design 34 (2004), no. 2, 183-190, DOI 10.1504/IJVD.2004.003901.

15 Szőke D, Lógó J, Optimum Suspension Parameters for Road Vehicles, VSDIA Conference (Budapest, Hungary, 2006), 2006, pp. 1-8.

16 Tettamanti T, Varga I, Distributed traffic control system based on model predictive control, Periodica Polytechnica - Civil Engineering 54 (2010), no. 1, 3-9, DOI 10.3311/pp.ci.2010-1.01.

17 Uys P. E., Els P. S., Thoresson M. J., Suspension settings for optimal ride comfort of off-road: vehicles traveling on roads with different roughness and speeds, Journal of Terramechanics 44 (2007), no. 2, 163-175, DOI 10.1016/j.jterra.2006.05.002. 